Barbara Börner-Westphal, (Berlin)

\title{
Sexus-Genus-Beziehungen im Hindi
}

\section{Summary}

In Hindi within the category of gender masculine and feminine can be distinguislsed. To a certain extent the opposition of malo and female is reflected in the opposition of masculine and feminine and sex can be identified by the gender of the noun as well as by secondary gender identifiers of the language. Yet, mainly in the case of nouns denoting trades and professions, posts, representatives of intellectual and political movements and relations between individuals the feminine opposites are missing. This gap in the language is bridged by the respective masculine plus a secondary gender identifier or by this masculine plus a lexical supplement and secondary gender identifiers. Yet, the above mentioned linguistic means that are used for denoting male and female individuals cannot be employed indiscriminately or regardless of the context. Certain sentence structures, too, rule out the use of certain means of the language for denoting gender explicitely.

1. Innerhalb der Kategorie des Genus werden im Hindi Maskulina und Feminina unterschieden. Das Neutrum, das z. B. im Sanskrit und auch in neuindoarischen Sprachon wie dem Marāthī und Gujarātī vorhanden ist, fehlt im Hindi.

Nur Substantive enthalten eine inhärente Genusangabo. Bei Personalpronomen (vah 'er, sie, es') 1 , Demonstrativpronomen (yah 'dieser, diese, dieses'; vah 'jener, jene, jenes') und Interrogativpronomen (kaun 'wer') dagegen fehlt sie.

Neben der inhärenten Genusangabe der Substantive können als sekundäre Genusanzeiger'2 bei Bezug auf ein Maskulinum oder Femininum auftreten: veränderliche Adjektive ${ }^{3}$ (acchā m., acchi f. 'gut') als Attribute oder Prädikativa, Possessivpronomen (merā m., meri f. 'mein'), Pronominaladjektive (aisä m., aisi f. 'solch ein'), Partizipien ( $\bar{a} t \bar{a}$ m., $\bar{a} t \bar{l}$ f. 'kommend'; $\bar{a} y \bar{a}$ m., $\bar{a} y \bar{\imath}$ f. 'gckonımen') als Bestandteile des Prädikats, als Attribute oder prädikative Attribute sowie als Bestandteile von Tempus- und Modusformen, einige Tempusformen sowie einige Modusformen (z. B. Präteritum, Konditional I u. a.), die neben der Kennzeichnung von Person und Numerus auch die entsprechenden sekundären Genusanzeiger bekommen müssen, der adjektivisehe Infinitiv (mujhe ek citthi likhni hai 'ich muß einen Brief schreiben') und die Genitivpostposition $k \vec{a}$, sofern sie eine Attribuierung bewirkt.

1 Fïr Demonstrationszwecke werden jeweils nur die Formen des Singulars angegeben.

2 Dieser Begriff wird hier im Gegensatz zum Begriff der inhüronten Genusangabe gebraucht. Sekundäre Genusanzeiger werden erst auf syntaktischer Ebene wirksam, sind also nicht Bestandteile von Wörterbuchformen.

${ }^{3}$ Für Demonstrationszweeke werden hier und in den folgenden Beispielen nur die maskulinen und femininen Formen im Singular angeführt. Die angegebenen einzelnen Hindiwörter stehen jeweils stellvertretend für eine ganze Gruppe. 
Die sekundäre Genusanzeige wird durch die in der Hindi-Syntax geltenden, teilweise recht komplizierton Kongruenzregehn realisiert. Allerdings soll schon hier daranf hingewiesen werden, daß der vollen Realisierung der selsundären Genusanzeige im Satz bestimmte Satzstrukturen entgegen wirken können, wovon in einem woiteren Teil dieses Aufsatzes zn sprechen sein wird.

2. In welchem Verhältnis stehen num Sexus und Genus, also natiurliches und grammatisches Geschlecht, im Hindi?

Als 'natürliches Geschlecht' oder 'Sexus' wird die biologische /ugehörigkeit zur' Gruppe der männlichen orler weiblichen Jabewesen bezeichmet. Es geht hier also um eine Erscheinmng mit konkretem Realitätsbezng. In Sprachen, die eine (ienusunterscheidung haben, kam das natürliche Geschlecht eines Lebewesens mittelhar durch das Genus seinen Ausdruck finden, d. h, cin männliches Lebewesen wird in der Regel durch ein Maskulinum, ein weibliches durch ein Femininum bezeichnet. Sexus-GenusBeziehungen können daher nur in einem bestimmten Teilbereich, nicht aber bei der generellen Einteilung der Substantive in Maskulina und Feminina im Hindi eine Rolle spielen, d. h. das (ienus einer Bezeichnung für ein I.ebewesen kamn einen Realitätsbezug haben und damit ein natiurliches benotat, ist sexusbezogen und damit, motiviert, während das (Xenus anderer Substantive willkiurlich mond mmotiviert ist.

Allerdings kann man daraus nicht den Schluß ziehen, daß es eine keine Ausnahme zulassende und in jedem Falle vorhersngbare Beziehung zwischen Sexus und Genus im Hindi gibt. Nicht nu im Hindi gibt es z. B. auch Feminina, die männliche Lebewesen bezeichnen können, und Maskulina, dic weibliche Lebewesen bezeichnen können." Grundsätalich kann man jedoch sagen, daß das Hindi, das die Möglichkeit der morphologischen und syntaktischen Kennzeichnung von maskulinen und femininen Substantiven hat, in diesem Rahnen bis zum gewissen Grade auch die Soxusopposition männlich-weiblich einordnet. Diese Einordnung erfolgt aber nicht durch eine scharfe Trennungslinie zwischen Maskulina und Feminina, da die sprachliche Zuordnung zur Gruppe der männlichen oder weiblichen Iebewesen weit über den Rahmen der Substantive, d. h. ïbcr die inhärente Genusangabe, hinausgeht.

Im folgenden soll nun das Problem der sprachlichen Kennzcichnung des Sexushezugs im Hindi für eine Gruppe von Icbewesen, nümlich für Personen, näher betrnchtet. werden, da das der Bereich ist, mit dem der Praktiker am ehesten konfrontiert wird. Die Frage der Sexus-Genus-Beziehnng fiir den Bereich der 'J'ies- und Jflanzenwelt ist ebenfalls nicht unproblematisch, eine umfassende Behandlung auch dieses Problems wiirde jedoch unseren abgesteckten Rahmen sprengen.

Bei den folgenden Ausführungen handelt es wich nicht um eine diachronische Untersuchung, sondern um Ergobnisse und Einsichten, die bei der Auswertung von hindisprachigem Material etwa der letzten zehn Jahre gewonnen worden sind.

3. Die sprachlichen Mittel, die das Hindi zur Kennzcichnung des Sexustezugs benutzt (immer im oben abgesteckten Rahmen auf Personen bezogen), lassen sich in drei große Gruppen einteilen :

1. lexikalische Mittel: Hier handelt es sich im wesentlichen um Substantive, bei denen

4 Das findet man vor allem im Bereich von 'I'ierbezeichmungen, z. R. lo mri f. 'Fuchs', billï f. 'Katze'. Aber nuch bei Personenbezeichnungen gibt es solcho F älle: baccā m. 'Kind' kann unterschiedslos für 'münnliches Kind' und 'weibliches lỉnd' be nutzt wesden, obwohl es die Form bacei f. 'Mädchen' ebenfalls gilst. 
die Zuordnung Maskulinum $\rightarrow$ männliche Person und Femininum $\rightarrow$ weibliche Person durch das inhärente Genus erfolgt und damit eindeutig und unumkehrbar ist (mātā f. 'Mutter'; pitā m. 'Vater').

2. grammatisch-syntaktische Mittel: Hier ist die Benutzung maskuliner Substantive zur Bezeichmung männlicher und weiblicher Personen einzuordnen. Die Kennzeichnung des Sexusbezugs erfolgt nur durch sekundäre Genusanzeiger im Satz (Bhärut $k e^{5}$ pradhānmantri Rājīv Gãndhi 'Indiens Premierminister Rajiv Gandhi'; Bhārat ki pradhānmantrī Indirā G'āndhì 'Indiens Premierministerin Indira Gandhi').

3. loxikalisch-grammatische Mittel: Hierunter fallen \%usätze wie muhilā, stri u. a. 'Frau', 'weiblich' zu (meist) maskulinen Substantiven und puruş 'Mann', 'männlich' ebenfalls zu maskulinen Substantiven, um damit die Sexusopposition anch auf sprachlicher Ehene explizit auszudriicken (mahila -mantri 'weiblicher Minister, eine Frau als Minister, Ministerin'; puruṣ mantri 'männlicher Minister, ein Mann als Minister, Minister').

Die Mittel der Gruppen 2. und 3. sind nicht gleichwertig und nicht unterschiedslos zu benutzen. Sie sind, wie noch zu erläutern sein wird, kontextabhängig.

Zu 3.1. Im Wortschatz des Hindi selbst lassen sich viele Beispiele fiir die Kennzeichnung der Opposition männlich-weiblich direkt durch Maskulina bzw. durch Feminina finden. Das trifft vor allem auf Wörter zu, die die Sexusopposition in ihrer allgemoinsten Form repräsontieren, diese Opposition mit dem zusätzlichen Merkmal 'verwandt' versehen, sie als zugehörig zu traditionellen Berufen und Kasten, zu Varnas u. a. kennzeichmen. Die Glieder der Opposition männlich-weiblich sind in der Sprache meist vollständig, d. h. paarig, vorhanden. ${ }^{6}$ Als Beispiele können genannt werden: puruș 'Mann' - strī 'Trau'; laṛkã 'Junge' - lurkki 'Mädchen'; bețā 'Sohn' beți 'Tochter'; dhobi 'Wäscher' - dhobin 'Wäscherin, Frau des Wäschers'; abhinetā 'Schauspieler' - abhinetrī 'Schauspielerin'; brähmaṇ 'Brahmano' - brāhmañi 'Brahmanin'.7 Vor allem bei Verwandtschaftsbezeichnungen und Kasten- und Berufsbezeichnungen ist das Maskulinum das Ausgangswort, von dem mit Hilfo bestimmter Suffixe feminine Substantive gebildet worden. Es gibt aber auch einzelne Beispiele dafiir, da B das Femininum der Ausgangspunkt für die maskuline Variante sein kann, z. B. bahan 'Schwestor' - bahanoi 'Mann der Schwester, Schwager'. Die entsprechenden Suffixe und Ableitungsregeln werden vor allem in hindisprachigen Grammatiken ausführlich angegeben.

Zu 3.2. Die sprachliche Kennzeichnung des Sexusbezugs allein durch das inhärente Genus oines Substantivs ist, wie schon erwähnt, nicht immer möglich. Vor allem bei

5 Neben der Bezeichnung der Mehrzahl hat im Hindi der J'lural u. a. auch die Funktion eines Höflichkeitsanzeigers, wodurch die Genuszuordnung natürlich nicht berïhmt wird, aber innerhalh der crfolgten Genuszuordnung in ler konkreten Äußerung ontsprechend dem Reulitätsbezug bzw. der Sprecherauffassung nach bestimmten Regeln der Singular oder Plural erscheinen kann: merā mitra (Sing.) 'mein Freund'; mere pitā ji (P'lural) 'mein Vater'. Die hier wirkenden Regeln sind nicht ganz identisch mit denen der Pluralbildung zur Bozeichnung der Mehrzahl.

6 Einzelne Ausnahmen gibt es auch hier: für sati 'eine Frau, die sich mit ihrem toten Mann verbrennen läßt' gibt $\mathrm{cs}$ in Indien keine münnlicho Variunte und damit auch kein entsprechendes maskulines Substantiv.

7 Unter dem Einflul3 dor noch zu behaudelnden Gruppe 3.3. werden jedoch auch zusätzliche Bezeichnungen für weibliche Personen gebildet. So gibt ea z. B. neben brähmaña auch brähman strī, neben mazdürin 'Arbeiterin' auch mazdür strī. 
Bezeichnungen für männliche und weibliche Vertreter bestimmter Berufe und Posten, bei der Bezeichnung von Vertretern bestimmter geistiger und politischer Strömungen, bei solchen Wörtern, die männliche und weibliche Personen in einer bestimmten, njcht verwandtschaftlichen Beziehung zu anderen Personen stehend charakterisieren, bei vielen Nomen agentis, die ebenfalls gleichzeitig Personenbeziehungen ausdriicken kömnen und bei Wörtel'n, die die Zugehörigkeit von Frauen und Männern zı Orten und ländern bezejchnen, gibt es neben solchen, die durch ein Maskulinum und ein Femininum vertreten sind, sehr viele, bei denen im lexikalischen Inventar ein Glied fehlt, und zwar meist das Femininum. Dadurch kann die Sexusopposition männlichweiblich ('Herr X ist Premierminister; Frau Y ist Premierministerin) als vollständige parige Genusopposition nicht mehr reprisentiert werden. ${ }^{8}$. as ist besonders häufig der Fall bei Bezeichnungen fiir Berufe und Posten, die erst in juingster Zeit zunehmend von Frauen besetzt werden. Die Ursachen dieser Erscheinung anfzudecken wäre demnach eine Sache der Soziolinguistik. J)ie sprachliche Realität muß aber von uns untersucht werden. Möglicherweise spielt hier auch der Einflul3 des Wnglischen eino Rolle, denn gerade unter diesen Wörtem befinden sich viele aus dem Englischen entlehnte.?

Ausgehend von der (möglichen) V'ollständigkeit oder Unvollständigkeit der beiden ('enusglieder Maskulinum - Femininum, die den Ausgangspunkt fïr die erforderlichen grammatisch-syntaktischen Strukturen bilden, kömnen die hier einzuordnenden sprachlichen Mittel in zwei Untergruppen unterteilt werden:

3.2.1. Diese Untergruppe bilden Substantive, die die Sexusopposition männlichweiblich jeweils auch durch ein Maskulinum bzw, ein Femininum kennzeichnen könnten. Im praktischen Sprachgebrauch jedoch wird das Vorhandensein des Femininums teilweise ignoriert und das Maskulinum wird zur Bezeichmung männlicher und weiblicher Personen benutzt, wobei der Sexusbezug im letzteren Falle mur noch auf der syntaktischen Bhene durch die anfangs erwähnten sekundären Gemusanzeiger gekemneichnet wird, es sei dem, dem stehen bestimmte Satzstrukturen im Wege. Das so benutzte Maskulinum hat jedoch in keinem Falle die morphologisehen Merkmale eines Femininums, es fehlt z. B. generell der Plural des Casus rectus. 10 /u diesen

8 \%war könnte im Hindi in vielen Fällen vor allem bei Entlehungen aus dem Sanskrit nach den Regeln der Sanskritgrammatik auch das Femininum gebildot werden, nber diese theoretische Möglichkeit wird bei dieser Gruppe von Würtern sclten genutzl. So fand sich in der benutzten Literatur kein Beleg etwa für mantrini o. ä. Andererseits gibt ro aber \%. I3. dio feminino Form adhikärinzi zu adhikäri, die aber nicht fïr dio lBedentung 'Benmte' benutz.t wird, sonlern nur für' eine andere Bedentung dieses Wortes, nümlich 'pine Fran, die Anspruch auf ... hat'.

(1)as Hindi folgt bei diesen Entlehnungen in wesentlichen dem englisehen Vorbild. Bei Vorhandensein nur eines Substantivs im Wnglischen als Bezeichnung für münnliche und weibliche Personen worden, wenn dieses ins Hindi ijbernommen wird, nuch mïnnliche und weibliche Tersonen danit bezeichnet, z. B. 'teacher' $\rightarrow$ licar m. 'Lchrer' und kontoxtnbhängig 'Lehrerin'. 13ei Vorhandensein von zwei verschiedenen Bezeichmungen für männliche und weibliche Jergonen im Englisehen wird das Wortpaar oft äbornommen, ж. B3. 'hero' $\rightarrow$ hïro m. 'Held', 'heroine' $\rightarrow$ hiroin f. 'Heldin'. Vereinzelt gibt es much feminine Neubildungen nach den Regeln der Findigrammatik: 'doctor' $\rightarrow$ dăkfar m. 'Arzt', dakkfurn i f. 'Ärztin'.

10 Auf das eingeschränkte Deklinationsschema von Maskulina, die zur Bezeichnung weib. licher Personen benutzt werden, weist auch V. P. LıperovskiJ, in: Iменниые иасти речи язына Хинди, Moskva 1978, S. 33 hin. 
Substantiven gehören u. a. sadasya m. 'Mitglied' - sadasyä f. 'weibliches Mitglied', eine Frau als Mitglied'; allhyakṣ 'Vorsitzender' - adhyakṣā f. 'Vorsitzende'; nirdeśak m. 'Direktor' - nirdesikā f. 'Direktorin'; khiläri m. Sportler' - khilärin f. 'Sportlerin' u. a. Möglich sind daher hei Aussagen über weibliche Personen folgende Satzstrukturen:

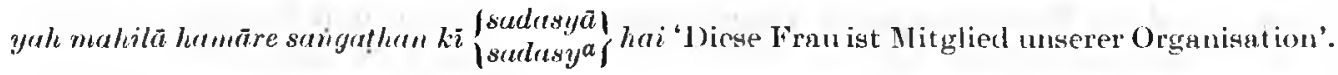

Auf männliche I'ersonen bezogen dagegen ist nur möglich:

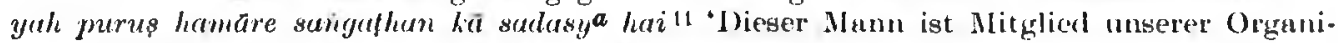
sation'.

Der Gebrauch des Maskulinums auch bei Vorhandensein eines Femininums, der bei sadusya und adhyakş besonders häufig zu beobachten ist, scheint nach unseren Ëin. schätzungen vom Individualstil abzuhängen. So benutzte z. B. die Autorin der Broschüre 'Die indische Frau auf dem Weg zum Fortschritt' 2 mit einer Ausnahme die vorhandenen Feminina. Ähnliches findet man auch in der Franenzeitschrift ' $T$ 'àmă', während Tageszeitungen wie die 'Nuvabhārut läims' dagegen vorwiegend die Maskulina für beide Sexus benutzen. Die Wahl des einen oder anderen Genus bei Vorhandensein des Femininums und des Maskulinums ist fiir diese Gruppe von Substantiven möglicherweise eine Stilfrage und eine Frage der 'Tendenz.

3.2.2. Die zweite Untergruppe wird ron maskulinen Substantiven gebildet, die je nach Kontext zur Bezejehmung männlicher oder weiblicher Personen benutzt werden können. Von einzelnen ungebräuchlichen Feminina abgesehen, deren Gebranch dem Individualstil zuzurechnen ist, 13 haben wir hier jeweils nur ein Genus, das beide Sexus vertreten mul.3. '/u diesen Maskulina gehören u. a.: mantri 'Minister' und alle Ablei-

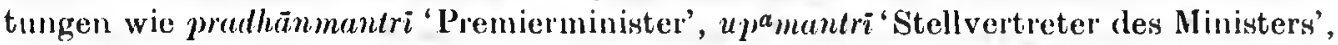
räj!lamantri 'Staatsminister', mukhyamantri 'Chefminister'; rāstrapati '(Staats-)Präsidont', pratinilhi 'Vertreter', nelä 'Führer', sāñsarl 'Parlamentsahgeordneter', rüjdüt 'Botschafter' usw.; alle Ableitungen mit dem Suffix -rä̀li, sofern sie P'ersonen bezeichnen, wie sumājvärli 'Sozialist' usw.; mitra, dost 'Freund', śatru 'Foind', śikär 'Opfer', nirmätä 'Schöpfer', mehmān 'Gast', samarlhak 'Unterstiitzer, Helfer' ; bhäraliy ${ }^{a}$ 'Inder', nägurik 'Biirger' u. v. a. Bei Bezug auf männliche Personen heißt es daher

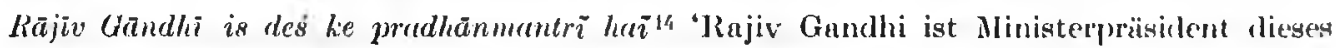
Landes'.

Bei Bezug auf eine weibliche Person jedoch muß es heißen

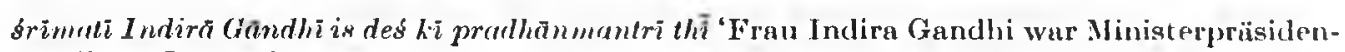
tin dieses Iandes'.

Hier und in anderen Kontexten wird dentlich, da 3 in Hindi, ähnlich wie z. B. im .Deutschen, „dns Femininum mit dem Merkmal 'weiblich' den Charakter eines markierten Gliedes mit einem auf dieses Merkmal eingeschränkten Geltungsbereich auf-

11 Der P'lural als Höflichkeitsanzeiger wurde hier außer acht gelassen, s. Fußnote 5.

12 R. l'ANIKAR, Bhäratīy a nărī pragrati ke path par, Dilī 1970.

${ }^{13}$ A. T. Aksenov z. B. untersuchte in einem Aufsatz, ob manträni f. als Konkurrenzform z.u mantri für die Bezeichnung weiblicher Minister angesehen werden kann. Er kum zu den Schluf, daß - von wenigen Ausnahmen abgesehen - die Tendenz eindeutig zur Benutzung des Maskulinums mantri auch für weibliche Personen geht. Vgl. A. T. Arcenor,

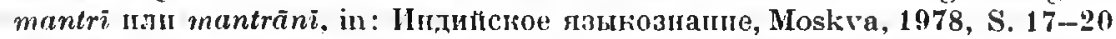

14 Der Plural steht als Höflichkeitsauzeiger. 
weist, während das Maskulinum offensichtlich den Charakter eines unmarkierten Gliedes mit umfassenderem Geltungsbereich erkennen läßt“..15 Die Sexusopposition hat in den genannten Fällen also keine volle lexikalische Entsprechung auf sprachlicher Ebene in Form eines Maskulinums und eines gebräuchlichen Femininums, sondern es wird nur ein Substantiv benutzt, das in seiner Wörterbuchform als Maskulinum geführt wirl, im aktuellen Kontext aber als Femininum (mit Einschränkungen) gebrancht werden kann, was sich jedoch nur im syntaktischen Umfeld zeigt. Das so benutate Wort zeigt, auch wenn es für weibliche Personen benutzt wird, ebenso wie die maskulinen Substantive der Lntergruppe 3.2.1. keinerlei für Feminina charakteristische morphologische Besonderheiten. Diese Maskulina sind also in gewisser Weise '"/witterwörter', die eben nur dadurch die entsprechende Rolle bei der Bezeichnung des weiblichen Teils der Sexusopposition übernehmen können, weil das Maskulinum weniger markiert in bezug auf das natiirliche Geschlecht zu sein scheint. Die Benutzung von Feminina zur Bezeichnung des männlichen Teils der Sexusopposition dagegen ist in der Regel nicht möglich.16

Da diese Frscheinung auf bestimmte Substantive und Kontexte beschränkt ist, zudem auch nur im Sexus-Genus-Bezug zutage tritt, sollte man u. E. nicht von einer 'Unterspïlung' der Kategorie des Genus im Hindi sprechen. ${ }^{17}$ Auch der Begriff 'common gender' ${ }^{9}$ oder 'обция poл' 19 ist nicht zutreffend, weil ja die Merkmale 'maskulin' und 'feminin' durchaus nuf bestimmten sprachlichen Ebenen realisiert werden. ${ }^{20}$ Das

15 h. F. Heldor.ph, W. Fı̈̈ma, W. Motsch, Grundzüge einer deulschen Grammatik, Berlin, 21984, S. 574. Mlit der geringen Sexusmarkierung der Maskulina kunn auch der Gebrunch des Plural il. für die Bezeichnung einer Gruppe aus münnlichen und weiblichen Personen (chăra 'Studenten') und für Paare (matä-pita 'Mutter und Vater', 'Eltern') im Hindi erklürt werden. Ebenfalls als l'lural m. werden in der Regel Komposita aus einem Maskulinum und einem Femininum behandelt, die eine Mischgruppe bezeichnen, unabhängig duvon, ob z. 13. - wie es bei bestimmten Kombinationen der Full ist - das letzte Glied das morphologische Kennzeichen eines Plural f, trïgt : chăra chriträè 'Studenten und Studentinuen', nar - nari 'Nünner und Frauen'. Allerdings koument es auch vor, dalj Kombinntionen wis yurd-yuvatiya 'junge Männer und Frauen', 'Jugendliche' ein Jrädikat im Plural f. zugeordnet wird, wälrend Attribute dagegen in Plural m. erscheinen. Hier zeigt sich die Schwierigkeit der grammatischen Zuordnung solcher Komposita. Im Gegensatz zu cinom Mnskulinum in der Pluralform scheint der Singular m. aber doch in der Regel stärker sexusbezogen zu seiu. Von einigen Ausnahmen ahgesehen sind dagegen Fentinina im Singular und Plural immer sexusbezogen, soweit sie I'ersonen bezeichnen. Andererseits hat das Hindi aber auch Mittel, um sprachlich einer 'Gruppe nur weiblicher Personen' eine 'Gruppo nur münnlicher l'ersonen' gegenüberzustellen, s. 3.3.

16 Von einem Muttersprachler wurde mir eine Ausnahme genunnt: nars 'Krankensehwe. ster', dus in der Form 'purus nars' als 'Krankenpfleger' benutzt werden kann.

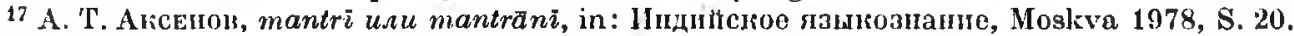

${ }^{18}$ A. Sharma, A basic Grammar of modern Hindi, New J)elhi ${ }^{3} 1975$, S. 31.

19 V. T. LIPFROVSKiJ, a. a. O., S. 34.

21) In diesem Zusammenhang mul3 man die Frage stollen, wie Lintragungen für solche Wörter in zweisprachigen Wörterbüchern crfolgen sollen. Die Kennzeichnung mantri m./f. z. 13. ist nicht korrekt, da der Benutzer daraus den falschen Schluß zichen kann, dal3 es auch den Plural f. geben müßte. Hilfreich wäre dio Kennzeichnung durch ein besonderes Symbol, was auf die Sonderstellung dieser Substantive hinweist. HindiGrammatiken behandeln das Problem nur sporadisch. Die als Standard-Hindi-Grammatik angesehene Basic Grammar of modern Hindi, Now Delhi ${ }^{3} 1975$, führt auf S. 31 zwei solcher' Substantive an, nümlich mamtri und mitra, während R. P. SIÑ HA in ' $S$ 'uddh

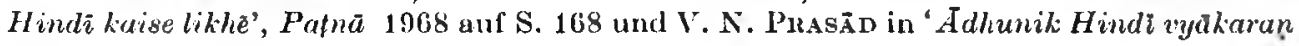


Maskulinum als Wörterbuchform ist bei den beschriebenen Fällen das in Bezug auf das Sexus weniger markierte Glied, es kann offenbar die Merkmale ' \pm männlich' und ' \pm weiblich' tragen, während bei Personenbezeichnungen das Femininum mit dem Sexusbezug '+ weiblich' markiert ist.

Zu 3.3. Bei den bisher erwähnten Beispielen zur sprachlichen Kennzeichnung des Sexusbezugs im Hindi ging es um die Darstellung solcher Sachverhalte wie 'er/sie ist Vorsitzender/Vorsitzende von ...', 'er/sie hat den Posten eines/einer ... inne' usw. Die Kennzeichnung des natürlichen Geschlechts ist hierbei nicht der Kern der Aussage. Sie ist zwar wichtig, bleibt aber gegenüber der Berufsbezeichnung u. a. sekundär. Es zeigte sich, daß das Hindi in solchen Fällen vielfach weibliche Personen durch Maskulina bezeichnet, der so entstandene Widerspruch zwischen Sexus und Genus jedoch auf syntaktischer Ebene soweit wie möglich gelöst wird.21

Boi der sprachlichen Wiedergabe des Sexusbezugs kann nun ein weiterer Fakt bedeutend werden: es kann erforderlich werden, nicht nur einen männlichen oder weiblichen Vertreter zu nennen, sondern die Opposition männlich-weiblich auch sprachlich als Opposition auszudrücken, wobei dann die Bezeichnung des natürlichen Geschlechts in den Vordergrund rückt. Das wäre etwa der Fall bei Aussagen wie 'sie war die erste Ministerpräsidentin der Welt (= sie war die erste Frau auf dem Posten ejnes Ministerpräsidenten)' oder 'der Prozentsatz der weiblichen Abgeordneten ist gering', also Kontexte, die sich deutlich von den o. g. unterscheiden. Das Hindi benutzt in diesen Fällen Zusätze wie mahilā, stri und ledi, um den Sexusbezug '+ weiblich' auszudrücken, und (meist) puruș, um den Bezug ' + männlich' auszudrïcken, wobei die Zusätze für '+ weiblich' mit Maskulina, seltener auch mit Feminina, die Zusätze für '+ männlich' jedoch nur mit Maskulina vorkommen.22 Die so gekennzeichneten Maskulina und Feminina gehören vorwiegend in die Gruppen 3.2.1. und 3.2.2., aber auch einzelne Substantive der Gruppe 3.1. werden so benutzt. Das Genusverhalten der gesamten Neubildung wird, wenn Grundwort und Zusatzwort im Genus nicht übereinstimmen, durch das Kusatzwort bestimmt: mahilä + pradhänmantrī m. $\rightarrow$ mahilä pradhänmantrī, sekundäre Genusanzeiger, die auf mahilā pradhānmantrī bezogen werden, müssen feminine Endungen bekommen, während die Neubildung selbst wiederum nicht die morphologischen Eigenschaften eines Femininums annimmt, sondern ebenso wie die für die Bezeichnung weiblicher Personen benutzten Maskulina der Gruppe 3.2. in Bezug auf die Deklination defektiv ist.

Der Sexusbezug zeigt sich hier nicht allein im aktuellen Kontext, sondern schon in der Wortkombination durch die Zusätze mahilā usw. und purus. Die so entstandenen Wortkombinationen sind aber ebenfalls keine Wörterbuchformen. Die Möglichkeit,

aur racnā', Patnā 1979 auf S. 148 immerhin 16 bzw. 19 solcher Wörter anführen, die sie als ' $u$ bhaylingi 'mit doppeltem Genus' bezeichnen. V. P. I.IPERovskus behandelt das Problem kurz in der schon zitierten Monographie auf den Seiten 30-34, konzentriert sich dabei jedoch auf den Plural $\mathrm{m}$. und auf die Möglichkeit lexikalischer Zusätze wie mahilä u. a., ohne auf konkrete syntaktische Konsequenzen hinzuweisen. Wir sehen es daher als unsere Aufgabe an, eine möglichst vollständlige Aufzäh. lung der betreffenden Substantive für Lehrzwecke zu erreichen. Die bisher erreichten Ergebnisse im Rahmen dieses Aufsatzes als Liste anzufügen ist aus J'Jatzgrïnden jedoch nicht möglich.

21 J3estimmte Einschränkungen, die wiederum von Satzstrukturen abhängig sind, werden Thema des zwciten Teils dieses Aufsatzes sein.

$22 \mathrm{Zu}$ einer Ausnahme a. Fußnote 16. 
mahilā usw. mit Maskulina zu kombinieren, weist wiederum auf die relative Sexusindifferenz der Maskulina bei bestimmten Personenbezeichnungen hin. ${ }^{23}$

Soll die Aussage daher eindentig sein, muß der Sexusbezug in bestimmten Kontexten durch die angeführten lexikalischen Zusätze angegeben werden, inshesondere auch dann, wenn maskuline Substantive, die auch bei der Gruppe 3.2. eine Rolle spielen, im Plural benutzt werden:

baink ke karmcāri hartāl karēge 'Die Angestellten der Bank (männliche und weibliche; nur münnliche?) werden streiken’

baink kì mahilā karmcāri hartäl karëgì 'Iie weiblichen Angestellten der Bank werden streiken'

baink ke purus karmcāri hartāl karẽge 'Die männlichen Angestellten der Bank werden streiken'

Ein ganz bestimmter Kontext könnte zwar dem Plural m. auch die Rolle der Bezeichnung einer Gruppe nur männlicher Personen zuweisen, dieser Plural jedoch kömnte u. U. wegen seiner auch verallgemeinernden Bedeutung mißgedeutet werden. Jer Zusatz von puruş zum Maskulinum macht auch hier den Sexusbezug eindeutig. Aucl der Singular m., der im allgemeinen eher als sexusbezogen empfunden wird, kann so markiert werden: puruş chätra 'mänulicher Student'. J)as Muster 'puruş+ Maskulinum' wird nun im aktuellen Sprachgebrauch auch auf solche Substantive iibertragen, die durch die Genusopposition schon die Sexusopposition ausdriicken könnten, d. h.es wird ein zusätzliches Glied 'mahilā +Femininum' geschaffen: mahilä + sadasyjā f. $\rightarrow$ mahilā sadasyā. Diese Wortverbindungen sind selbstverstiandlich echte Feminina mit vollem Deklinationsschema. Wir halten diese Erscheinung nicht vorwiegend für ein Ergebnis von außersprachlichen Einfliissen, ${ }^{2}$ sondern einmal für das innersprachlicho Bestrohen, dem Systemzwang folgend bestimmte Jeerstellen stets zu hesetzen, und zum anderen für eine typische Erscheinung des Hindi, vieles doppelt zu charakterisieren.

So gibt es für bestimmte Substantive im Rahmen der hier behandelten Problematik folgendo Kombinationsmöglichkeiten (dargestellt am Beispiel sadasya - sadasyā):

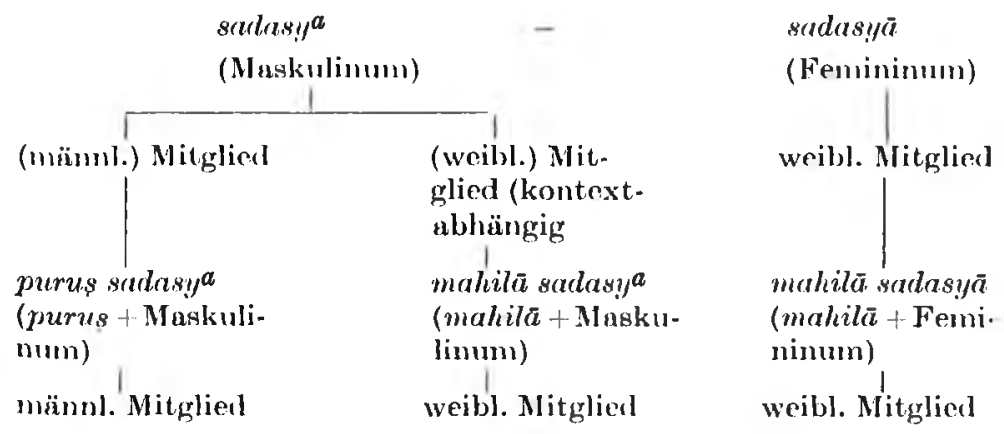

Wenn man die entsprechenden Kontoxte betrachtet, können jedoch mahilä sadasya/ mahilä sadasyjā und das entsprechende Fenininum sadusyā nicht unterschiedslos benutzt werden. Die Utber'setzung des Satzes

${ }^{23}$ Etwas anders verhalten sich z. B. 'Tierbezeichnungen, wo ohne weiteres Zusätzo wie nar 'Männchen, männliches Tier' mit Feminina kombiniert werden können.

${ }^{24}$ Vgl. A.T. Аксенон, Аналитический способ өыразяения рода имеи суияствителных в современиом Xинди, in: Јитийское пзиознанпе, Moskva, 1978, S. 14-16. 
'Sie wurde Mitglied der Kongrelspartei' ist: vah kāngres ki sadasya/sadasyā banī jedoch nicht: "vah kāngres ki mahilä sadasya/mahilā sadasyā banī.

In der Utbersetzung des Satzes

'Sie war das erste weibliche Mitglied des Parlaments' dagegen muß stehen : vah sañsad kì pahli mahilä sadasya/mahilā sarlasyā thì.

Gerade in diesen Sätzen zeigt sich deutlich der unterschiedliche Kontext für die sprachlichen Mittel der Gruppen 3.2. und 3.3.

4. Aus den bisherigen Ausführungen wurdo deutlich, da $\beta$ das Hindi mit den genannten lexikalischen, grammatisch-syntaktischen und lexikalisch-grammatischen Mitteln die Möglichkeit hat, bei Personenbezeichnungen den Sexusbezug sprachlich zu kennzeichnen. Der außersprachliche Kontext und die im sprachlichen System enthaltenen Mittel steuern dabei gemeinsam die Wahl der erforderlichen sprachlichen Einheiten zur Realisierung einer Aussage. Die bisher behandelten, im Sprachsystem des Hindi angelegten Möglichkeiten, mit unterschiedlichen Mitteln den Sexusbezug auszudrücken, werden jedoch im praktischen Sprachgebrauch durch einige syntaktische Strukturen blockiert, was dazu führen kann, da B der Sexusbezug in diesen Sätzen auch nicht mehr durch sekundäre Genusanzeiger ausgedrückt werden kann. Dieses Problem wird Gegenstand des zweiten Teils dieses Aufsatzes sein.

\author{
Abkürzungen \\ In. - maskulin \\ f. - feminin \\ männl. - แn̈̈nulich \\ weibl. - weiblich
}

Dr. Barbara Börner-Westrhal, Humboldt.Universität zu Berlin, Selition Asienwissenschaften, Bereich Südasien 